OPEN ACCESS

Edited by:

Vipin Srivastava,

University of Hyderabad, India

Reviewed by:

José Manuel Reales,

National University of Distance

Education (UNED), Spain

Madhura Ingalhalikar,

Symbiosis International University,

India

*Correspondence: Amitabha Ghosh amitabhaghosh269@gmail.com

Raju Surampudi Bapi raju.bapi@iiit.ac.in

${ }^{\dagger}$ These authors have contributed equally to this work and share first authorship

Specialty section: This article was submitted to Cognitive Neuroscience,

a section of the journal

Frontiers in Human Neuroscience

Received: 22 June 2021 Accepted: 12 October 2021 Published: 12 November 2021

Citation:

Dwivedi M, Dubey N, Pansari AJ, Bapi RS, Das M, Guha M, Banerjee R,

Pramanick G, Basu J and Ghosh A (2021) Effects of Meditation on

Structural Changes of the Brain in Patients With Mild Cognitive Impairment or Alzheimer's Disease

Dementia.

Front. Hum. Neurosci. 15:728993. doi: 10.3389/fnhum.2021.728993

\section{Effects of Meditation on Structural Changes of the Brain in Patients With Mild Cognitive Impairment or Alzheimer's Disease Dementia}

\author{
Madhukar Dwivedi't, Neha Dubey2,3t, Aditya Jain Pansari', Raju Surampudi Bapi ${ }^{1 *}$, \\ Meghoranjani Das², Maushumi Guha ${ }^{4}$, Rahul Banerjee ${ }^{5}$, Gobinda Pramanick ${ }^{6}$, \\ Jayanti Basu ${ }^{3}$ and Amitabha Ghosh ${ }^{2 *}$ \\ ${ }^{1}$ Cognitive Science Lab, International Institute of Information Technology, Hyderabad, India, ${ }^{2}$ Department of Neurology, \\ Apollo Gleneagles Hospital, Kolkata, India, ${ }^{3}$ Department of Applied Psychology, University of Calcutta, Kolkata, India, \\ ${ }^{4}$ Department of Philosophy, Jadavpur University, Kolkata, India, ${ }^{5}$ Crystallography and Molecular Biology Division, Saha \\ Institute of Nuclear Physics, Kolkata, India, ${ }^{6}$ Department of Radiology, Apollo Gleneagles Hospital, Kolkata, India
}

Previous cross-sectional studies reported positive effects of meditation on the brain areas related to attention and executive function in the healthy elderly population. Effects of long-term regular meditation in persons with mild cognitive impairment $(\mathrm{MCl})$ and Alzheimer's disease dementia (AD) have rarely been studied. In this study, we explored changes in cortical thickness and gray matter volume in meditation-naïve persons with $\mathrm{MCl}$ or mild $\mathrm{AD}$ after long-term meditation intervention. $\mathrm{MCl}$ or mild $\mathrm{AD}$ patients underwent detailed clinical and neuropsychological assessment and were assigned into meditation or non-meditation groups. High resolution T1-weighted magnetic resonance images (MRI) were acquired at baseline and after 6 months. Longitudinal symmetrized percentage changes (SPC) in cortical thickness and gray matter volume were estimated. Left caudal middle frontal, left rostral middle frontal, left superior parietal, right lateral orbitofrontal, and right superior frontal cortices showed changes in both cortical thickness and gray matter volume; the left paracentral cortex showed changes in cortical thickness; the left lateral occipital, left superior frontal, left banks of the superior temporal sulcus (bankssts), and left medial orbitofrontal cortices showed changes in gray matter volume. All these areas exhibited significantly higher SPC values in meditators as compared to non-meditators. Conversely, the left lateral occipital, and right posterior cingulate cortices showed significantly lower SPC values for cortical thickness in the meditators. In hippocampal subfields analysis, we observed significantly higher SPC in gray matter volume of the left CA1, molecular layer HP, and CA3 with a trend for increased gray matter volume in most other areas. No significant changes were found for the hippocampal subfields in the right hemisphere. Analysis of the subcortical structures revealed significantly increased volume in the right thalamus in the meditation group. 
The results of the study point out that long-term meditation practice in persons with $\mathrm{MCl}$ or mild $\mathrm{AD}$ leads to salutary changes in cortical thickness and gray matter volumes. Most of these changes were observed in the brain areas related to executive control and memory that are prominently at risk in neurodegenerative diseases.

Keywords: meditation, mild cognitive impairment, Alzheimer's disease, cortical thickness, gray matter volume, imaging, neuropsychology

\section{INTRODUCTION}

Alzheimer's disease is a clinico-pathological continuum caused by abnormal deposition of misfolded beta-amyloid and tau proteins with progressive breakdown along the core neurocognitive networks in the brain. Clinical progression comes much later and proceeds from a preclinical state, through mild cognitive impairment to dementia (Petersen et al., 2001; Albert et al., 2011; Dipasquale and Cercignani, 2016; Jack et al., 2018). Annually, around $10-15 \%$ of patients with MCI could convert to Alzheimer's disease dementia (hitherto referred as "AD"), Petersen et al. (2001) and over half of all converters are likely to do so within 5 years (Gauthier et al., 2006). Despite significant conceptual advances, the absence of effective disease-modifying drugs is frustrating and is pushing researchers to find alternative options to delay disease progression.

Of late, studies on meditation have consistently demonstrated structural and functional changes in the brain, including in gray matter volume, cortical thickness, and white matter tracts (Fox et al., 2014), regional cerebral blood flow (Newberg et al., 2010), and functional activity (Fox et al., 2016). During common meditation techniques, the meditator actively attends to breath, sensations, mantras, sounds or objects in what has been described as "focused attention" tasks, or to the rise and fall of thoughts and sensations in "open monitoring" tasks (Lutz et al., 2008). The default component is passive mind wandering, from where the meditator has to repeatedly turn the attention back to the task at hand. Other techniques such as loving-kindness meditation and meditation on peace, where faces, thoughts, or scenes are visualized, respectively, could be considered variants of "focused attention" meditation.

Hasenkamp and Barsalou (2012) demonstrated the effects of meditation on the core neurocognitive networks in healthy meditators. In healthy subjects, the activities of the default mode network (DMN) and the central executive network (CEN) are anti-correlated, activation of one being coupled with the deactivation of the other, for example, when a healthy person switches from self-referential thinking or mind wandering to a goal-directed focused task, or vice versa. The salience network (SN) recognizes the immediate salience of the task at hand and executes this switch (Seeley et al., 2007; Sridharan et al., 2008). Hasenkamp and Barsalou (2012) demonstrated that the same coordination between the three networks takes place during focused meditation. As a meditator moves from mind wandering to awareness of mind-wandering to switching back to focused breathing, there is corresponding activation of the DMN, SN, and CEN, respectively, until the cycle repeats. Indeed, meditation can be considered an exercise in cognitive control that has to pass through repeated phases of anti-correlated activation and deactivation of reciprocal neurocognitive networks.

A meta-analysis of studies in healthy meditators (Fox et al., 2014) showed that the most dependable morphometric differences in favor of meditation were found in the left rostrolateral prefrontal cortex (BA 10), anterior and mid-cingulate cortex, anterior insula, primary/secondary somatomotor cortices, left temporal gyrus and hippocampus. The meta-analysis, however, largely depended on studies on young to middle-aged meditators and did not include studies on patients with cognitive impairment. Reports on brain changes in elderly meditators are sparse (Luders et al., 2015; Chételat et al., 2017) and longitudinal studies in this cohort are lacking.

Few researchers have studied the effect of meditation (or mindfulness) on structural or functional changes in the brain in patients with MCI or AD (Newberg et al., 2010; Wells et al., 2013a,b; Yang et al., 2016; Fam et al., 2020; Yu et al., 2021). Morphometric studies are even rarer (Wells et al., 2013b; Yang et al., 2016; Yu et al., 2021) and none included patients with mild AD. In the structural MRI component of their studies, both Wells et al. (2013b) and Yang et al. (2016) assessed gray matter volume but not cortical thickness, whereas Yang et al. (2016) especially focused on the dorsal anterior cingulate cortex and bilateral hippocampi. Further, most of the studies so far have used various mind-body exercises rather than meditation alone. The Mindfulness-Based Stress Reduction (MBSR) protocol (Kabat-Zinn, 1990) followed by Wells et al. (2013b) includes body movements as one of the components. Yang et al. (2016) used Kirtan Kriya protocol that includes chanting of a mantra and finger movements during meditation. Movements also formed an important part of the protocol followed by Yu et al. (2021). All of these make it difficult to interpret the contribution of each component of the meditation protocol to the eventual structural or functional alterations in the brain. Moreover, Yu et al. (2021) used a low-intensity meditation protocol whereby patients were required to come weekly for the first 3 months and then monthly for the next 6 months. Follow-up assessments also varied between studies from 8 weeks to 9 months (Wells et al., 2013b; Yang et al., 2016; Yu et al., 2021).

Several unexplored or underexplored avenues therefore remain. For example, what would be morphometric brain changes following daily meditation if performed over a long term in both MCI and AD subjects? What would such changes be if only a silent, sitting meditation protocol is followed, without involving movements or vocalization in the process? Does repeated top-down modulation of behavior that is inherent 
in meditation practice bolster the regions of the brain that relate to executive control?

In this study, we try to address some of these issues as we investigate the impact of long-term daily silent, sitting meditation on brain gray matter volume and cortical thickness in MCI and mild $\mathrm{AD}$ patients. Given the limited data available in the literature on this topic, we plan to approach our query using whole-brain analysis for morphometric changes including the hippocampal subfields and other subcortical structures rather than focusing on a priori regions of interest. We hypothesize that long-term meditation in patients with MCI and mild $\mathrm{AD}$ will benefit brain areas that underlie attention and executive control. We also hypothesize that long-term meditation in these patients will not benefit the more severely affected posterior areas of the brain such as the posterior cingulate cortex (PCC).

\section{MATERIALS AND METHODS}

\section{Participants}

In this hospital-based longitudinal study, patients who met the clinical criteria of either amnestic MCI (Winblad et al., 2004; Albert et al., 2011) or probable AD (Jack et al., 2011) were recruited between November 2018 and March 2020, based on the following criteria: age between 45 and 70 years; formal education up to at least 8th grade; no psychotherapy for at least 2 months before recruitment; medication prescribed for dementia or MCI should have been at a constant dose of at least 4 weeks before recruitment. Patients also needed to have a global Clinical Dementia Rating (CDR) score between 0.5 and 1 (Morris, 1993) and a Fazekas score of between 0 and 2 on their screening MRI scans (Fazekas et al., 1987). Subjects were excluded from the study if they had been practicing any form of meditation or mindfulness procedures, had unstable medical conditions, active or chronic major organ disease, strokes or traumatic brain injury or seizures within 12 months or with residual disability, history of multiple strokes or transient ischemic attacks (TIAs), obstructive sleep apnea, psychiatric disorders other than mild to moderate anxiety or depression, had been on newly prescribed psychotropic drugs or underwent dose change within the last 4 weeks. All participants were required to have a caregiver accompanying them.

Detailed information of the study was provided to each patient and written informed consent was obtained. The Institutional Ethics Committee of Apollo Gleneagles Hospital, Kolkata, India cleared the study.

Forty-eight eligible patients consented to participate in the study. Recruited subjects were assigned into a meditation group and control groups by a convenience sampling method. Control groups in turn were subdivided into an active control (nonmeditation-focused task) group and a treatment-as-usual (TAU) group. Treatment as usual was kept constant for all groups and included anti-dementia medicines where indicated, avoidance of drugs that could exacerbate cognitive impairment, correction of reversible risk factors, and advice on lifestyle measures.
All recruited participants underwent a detailed assessment including clinical history, physical examination, and neuropsychological assessments by a cognitive neurologist and a clinical neuropsychologist. The neuropsychological tests were selected on the basis of the commonly reported changes in previous meditation studies and included the Montreal Cognitive Assessment (MOCA) (Nasreddine et al., 2005), CDR sum of boxes score (CDR-SB) (Morris, 1993; O'Bryant et al., 2008), digit span forward and backward, Trail Making Test (TMT)-black and white A and B (Kim et al., 2014), and the CERAD word list memory test (Morris et al., 1989). All patients underwent brain imaging. The researchers involved in MRI image acquisition (GP) and image analyses (MD, AJP, RSB) were blinded to the assigned groups as well as to the clinical and neuropsychological findings.

\section{Intervention Protocols}

Participants in the meditation group completed training sessions over 2 weeks, under the direct guidance of a trainer. Most of the meditative procedures were culled from acclaimed classical sources such as the Patanjali Yoga Sutras (Vivekananda, 2001) and the Path of Purification (Buddhaghosa and Nanamoli, 2003; Banerjee and Chatterjee, 2018). Out of several procedures, a subset of four meditation procedures was selected, namely, full-body relaxation technique, meditation on peace, witnessing the breath, and witnessing of thoughts.

After a detailed explanation of the process and any required clarification, the trainer conducted a "guided meditation" wherein step-by-step instruction was provided to the patient to induce the meditative state. Each meditation protocol was treated as an independent unit and generally, one protocol was taught to the patient per class on a one-to-one basis. After four sessions over a 2 -week period, a handout was provided to the patient describing the procedures in detail and a CD recording of the guided meditation provided for practice at home. Each session of guided meditation lasts about $30 \mathrm{~min}$ (1 $\mathrm{min}$ to settle down, 7 min per technique including around $10 \mathrm{~s}$ of transition time between each technique and 1 min to come out of meditation), which the patient was required to practice daily for the next 6 months.

\section{- Full Body Relaxation Technique}

Although this technique may not be considered meditation proper, it nevertheless precedes every meditation session. The meditator systematically surveys the entire body beginning with the feet and traveling to the head, consciously relaxing all muscular and nervous tension.

\section{- Meditation on Peace}

There are many variants to the meditation on peace. The procedure, which we have adapted for this study, has been simplified to enable all to perform. In this, the subject is encouraged to visualize a scene or suitably personalize a moment from the meditator's past, which is experienced as being calm and tranquil and can evoke a feeling of peace. The meditator is encouraged to self-assert this feeling of peace during this visualization. 
- Witnessing the Breath

In this, the subject is trained to concentrate on the natural and spontaneous flow of breath, without actively engaging in the process of inhalation or exhalation. The meditator gradually focuses the attention from chest movements to the nose to the nostrils and finally to a small point just below the nostrils where the out-breath brushes the upper lip on its way out. After anchoring attention to the breath, the meditator directs the attention to the various characteristics of the breath, including the in-breath and out-breath, their differences in temperature, and to changes in the length and rhythm of the breath. When extraneous thoughts arise, the meditator, on acknowledging the same, does not follow the thought but gently redirects the attention back to the breath.

- Witnessing of Thoughts

The subject is trained to observe the flow of thoughts in the attitude of a witness. This attitude implies an even-minded, detached observation of the flow of thoughts without regard to their specific content. The meditator is advised not to attempt to block the thoughts when they arise, nor to cling on to them but to simply observe how they appear and disappear spontaneously, and thereby acknowledge their transience. There must be clear apprehension of the thoughts and the meditator should ensure that she does not become drowsy or fall asleep.

Patients in the non-meditation-focused task group were given coloring books to work on for around $30 \mathrm{~min}$ daily for the next 6 months. When one set of coloring books was completed, another was provided to the patient. Patients were encouraged to choose their own colors and be imaginative.

Participants in both the meditation as well as the nonmeditation focused task groups were provided with logbooks and encouraged to document their time logs. Regular follow-up phone calls were made to participants to check for compliance and to discuss issues related to performance, if applicable. Participants were also encouraged to contact the research team for any queries.

\section{MR Image Acquisition}

High-resolution T1-weighted (T1-w) structural MRI scans were acquired using a $3 \mathrm{~T}$ Philips Ingenia MRI scanner with a standard multi-channel head coil. Foam pads were used to minimize head movements and facilitate comfortable scan procedures. For morphometric analysis, three-dimensional T1-weighted Magnetization Prepared Rapid Gradient Echo (MPRAGE) pulse sequencing was used. The whole-brain 3D T1weighted sequences were acquired with the following parameters: sagittal, the matrix size $=236 \times 236$, number of slices in each volume $=200,1 \times 1 \mathrm{~mm}^{2}$ in-plane resolution, slice thickness $=1.1 \mathrm{~mm}$, Repetition Time $(\mathrm{TR})=6.8 \mathrm{~ms}$, Echo time $(\mathrm{TE})=3.2 \mathrm{~ms}$, flip angle $=8^{\circ}$. The images were reconstructed and manually checked for major artifacts (e.g., motion, ringing, warp around) before further preprocessing.

\section{Follow-up Assessment}

By the end of 6 months of practice, 15 out of the 48 recruited patients $(31 \%)$ had dropped out from the study, most of them doing so within the first 3 months. Another patient who developed early Parkinsonian symptoms was excluded from the rest of the study. Given the variability in visit timings because of restrictions during the Covid-19 pandemic, the second assessment took place at a median of 6.7 months (Q1, Q3: 6.2, 7.5 months, respectively). Out of the 32 patients who completed their second neuropsychological evaluation, 22 participants (11 in the meditation group, five in non-meditation focused task group, six in the TAU group) could complete their second MRI scanning, the rest of the participants being reluctant to undergo MRI scanning during the pandemic. Given the small number of imaged patients in the focused task group and the TAU group, we combined them into one "non-meditation group" for the analyses of imaging data.

\section{Image Processing}

All high-resolution T1-w structural images were processed using publicly available Freesurfer software. Images were automatically processed with the processing pipeline related to the longitudinal stream in Freesurfer (Reuter et al., 2012). All scans from two time points (at time point 1 - the base scan and at time point 2 - the repeat scan) were first pre-processed independently with the cross-sectional stream. We have used the Recon-all stream for volumetric segmentation, which includes motion correction, skull stripping, intensity normalization, automated Talairach transformation, gray/white matter tessellation, and topology correction (Fischl et al., 2001; Ségonne et al., 2007). A base template was created for each participant from both the scans (at two-time points), which helps in the initial segmentation and surface reconstruction for structural data. For unbiased analysis in the longitudinal stream, measurements at the two-time points were registered to the base template. All segmented and surface reconstructed data were manually checked for segmentation accuracy for each time point. This preprocessing pipeline is widely used among researchers and provides high reliability and statistical power for structural data (Reuter et al., 2012). Freesurfer uses Desikan-Killiany Atlas (Desikan et al., 2006) to segment the cortex into 31 distinct cortical regions in each hemisphere based on the sulcal and gyral anatomy of the brain.

\section{Statistical Analysis}

Statistical analysis was designed to take into account the variable time lags between visits when interpreting the structural brain changes and neuropsychological test scores. This was done by calculating the symmetrized percentage change (SPC) values for each test. SPC is defined as the rate of change with respect to the average values/thickness/volume [i.e., $100 \times$ (annualized rate between time point 1 and time point 2)/average] (Reuter et al., 2012; Pegueroles et al., 2017). SPC can therefore be used to compare longitudinal differences between subjects even with variable inter time point intervals.

Symmetrized percentage change

$$
=100 * \frac{(t p 1-t p 0)}{\text { average }(t p 0, t p 1) * \text { time_difference }(t p 0, t p 1)}
$$

Where, $\operatorname{tp} 0 \rightarrow$ measure at baseline

tp $1 \rightarrow$ measure at final time point 
time_difference $(\mathrm{tp} 0, \mathrm{tp} 1) \rightarrow$ the time elapsed (in years) between the scans tp 0 and tp 1

To investigate the differences in different neuropsychological test sores among the three groups, Kruskal-Wallis nonparametric analysis of variance (ANOVA) was performed, as the sample size was small.

To investigate the changes in cortical thickness and gray matter volume, we performed two different types of statistical analysis, namely, two-stage regions of interest (ROI)-based analysis, and two-stage voxel-based analysis. In the first stage, we reduced the longitudinal data into a single statistic for each subject using SPC. In the second stage, we compared that single statistic across different groups. To observe any significant changes in 31 predefined ROIs in ROI-based analysis between the meditation and the non-meditation groups, a non-parametric independent sample $t$-test (Mann-Whitney $U$-test) was performed in JASP version 0.14.1 (JASP Team, 2020).

For hippocampal subfields and other subcortical structures such as the cerebellum, thalamus, caudate, putamen, pallidum, and amygdala, SPC in gray matter volume values was estimated for each region. For hippocampal subfields analysis, we normalized each subfield volume with its estimated total intracranial volume (eTIV).

Volume $(\text { normalized })_{i}=$

$$
\text { Volume (observed })_{i} * \frac{T I V_{m}}{T I V_{i}} ; \text { (Du et al., 2007). }
$$

Where, $\operatorname{TIV}_{\mathrm{m}} \rightarrow$ mean total intracranial volume for all subjects $\mathrm{TIV}_{\mathrm{i}} \rightarrow$ total intracranial volume of the ith subject

TABLE 1 | Demographics of participants who completed their neuropsychological assessments at baselines and follow-up.

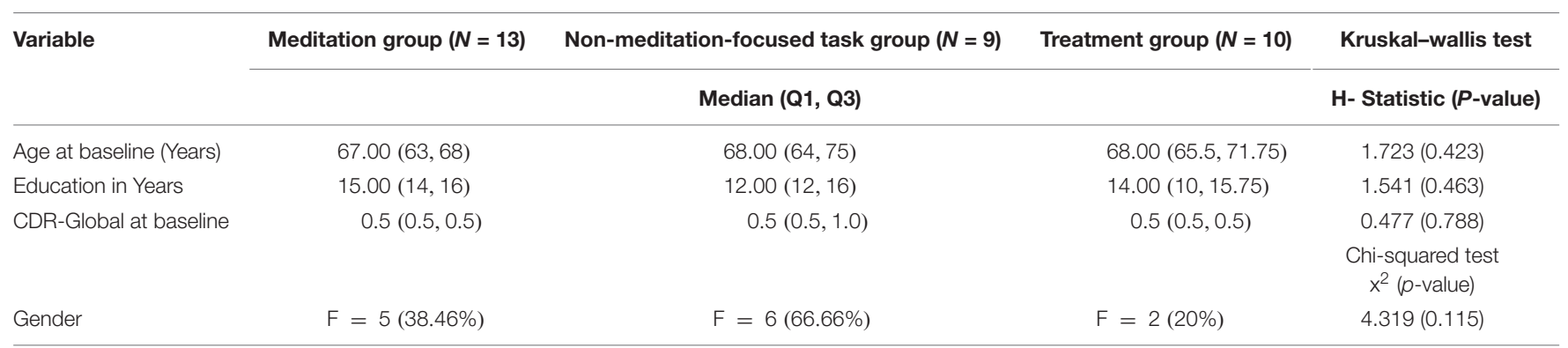

Gender: "F" represents the number of female subjects in the respective groups.

TABLE 2 | Neuropsychological scores (median value) from baseline to follow-up and "Median (Q1, Q3)," "H-statistic," and "P-value".

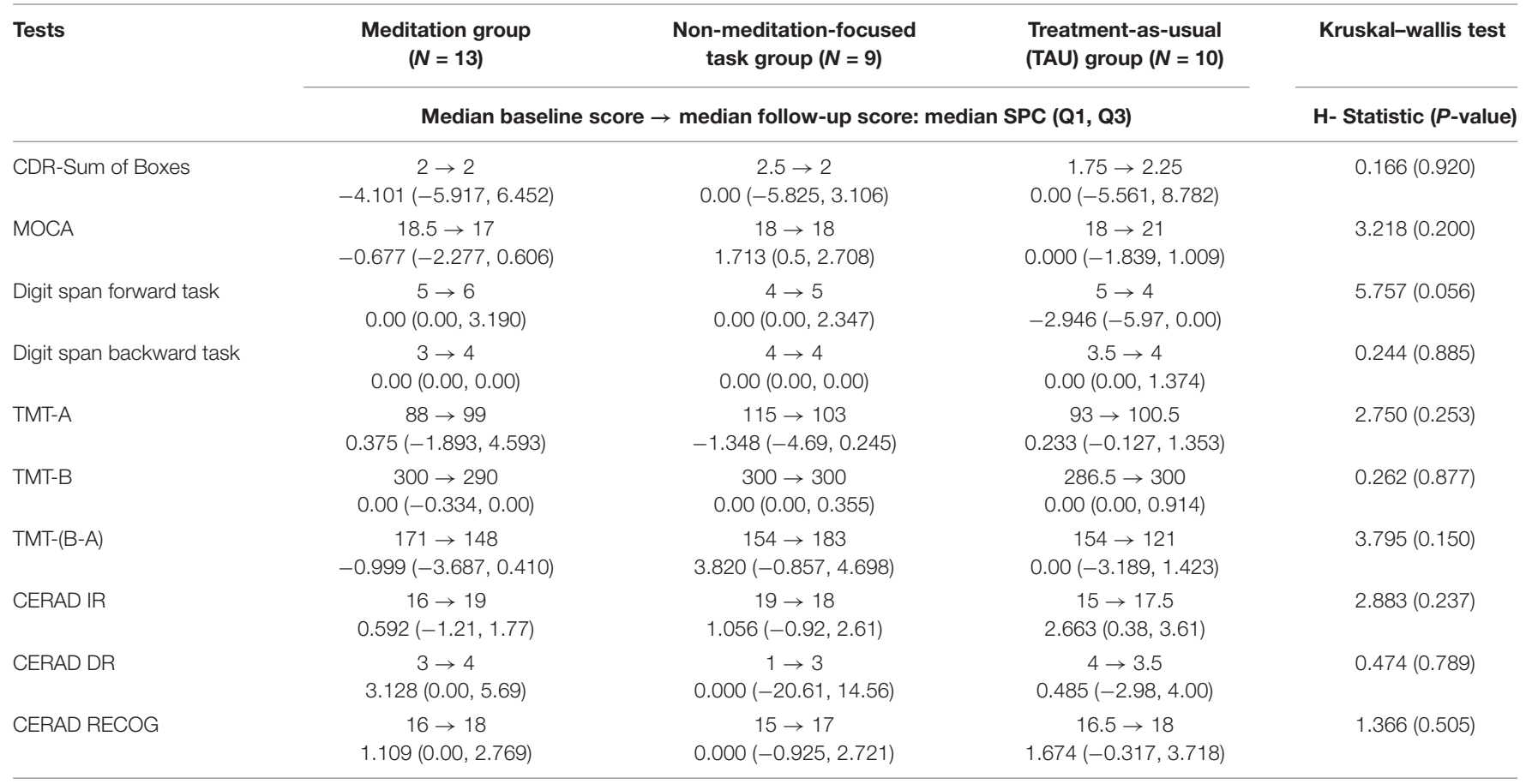

MOCA, Montreal Cognitive Assessment; TMT, Trail Making Task; CERAD, Consortium to Establish a Registry for Alzheimer's Disease; CERAD (IR), (DR), (RECOG), Immediate Recall, Delayed Recall, Recognition. 
An independent sample non-parametric $t$-test (MannWhitney $U$-test) was used to assess the significant gray matter volume changes between the meditation and nonmeditation groups for subcortical structures including all the hippocampal subfields.

Voxel-based statistical analysis for imaging data was performed using Freesurfer's built-in general linear model (GLM) tool - Query Design Estimate Contrast (QDEC) ${ }^{1}$. To investigate changes in the cortical thickness and gray matter volume, a longitudinal two-stage voxel-wise whole-brain GLM for both hemispheres was applied. The GLM model compared the SPC values in cortical thickness and gray matter volume between meditation and non-meditation groups. In the GLM model, age, gender, and education were treated as nuisance variables and correction for multiple comparisons was performed in all the analyses. We used the following criterion for considering a region to be significantly changed over time: the cluster should have a corrected false discovery rate (FDR) (Benjamini and Hochberg,

${ }^{1}$ https://surfer.nmr.mgh.harvard.edu/fswiki/FsTutorial/QdecGroupAnalysis_ freeview
1995) value of $p<0.05$ and the total size of all the clusters for a given ROI should be more than $50 \mathrm{~mm}^{2}$. We calculated the effect size for each ROI which showed any significant change between meditation and non-meditation groups. To compare the between-group differences for all measures, we used the nonparametric Mann-Whitney $U$-test. Rank biserial correlation effect size with a $95 \%$ confidence interval was calculated.

\section{RESULTS}

\section{Neuropsychological Data}

Table 1 shows the demographics and baseline global CDR scores of the 32 subjects who completed their second neuropsychological assessment. Baseline scores of age, gender, education, and global CDR scores did not differ significantly between the three groups.

None of the neuropsychological test scores differed significantly between the three groups (Table 2).

For the 22 subjects used in MRI analysis, baseline scores of age, gender, education, and global CDR scores did not differ

TABLE 3 | Demographics of participants who finished their baseline and follow-up neuroimaging scans.

\begin{tabular}{|c|c|c|c|}
\hline \multirow[t]{2}{*}{ Variable } & $\begin{array}{l}\text { Meditation group } \\
\qquad(N=11)\end{array}$ & $\begin{array}{l}\text { Non-meditation group (Focused task+treatment) } \\
\qquad(N=11)\end{array}$ & \multirow[t]{2}{*}{ Mann-Whitney $U$ test } \\
\hline & \multicolumn{2}{|r|}{ Median (Q1, Q3) } & \\
\hline Age at baseline (Years) & $67.00(64,68)$ & $71.00(59.5,72.5)$ & $41.5(0.223)$ \\
\hline Education in Years & $15.00(13,16)$ & $14.00(12,15.5)$ & $71.5(0.484)$ \\
\hline CDR-Global at baseline & $0.50(0.5,0.75)$ & $0.50(0.5,0.5)$ & $66.0(0.651)$ \\
\hline
\end{tabular}

Gender: "F" represents the number of female subjects in the respective groups.

TABLE 4 | Cortical thickness and gray matter volume changes in ROI-based analysis between the meditation and non-meditation groups in both hemispheres.

\begin{tabular}{|c|c|c|c|}
\hline ROI name & Meditation group $(N=11)$ & Non-meditation group (Focused task+treatment) $(N=11)$ & Mann-Whitney $U$ test \\
\hline \multicolumn{4}{|c|}{ Cortical thickness (Meditation > Non-meditation) } \\
\hline Left rostral middle frontal & $5.23(1.32,17.97)$ & $0.942(-5.93,3.95)$ & $93(0.034,0.53)$ \\
\hline \multicolumn{4}{|c|}{ Cortical thickness (Meditation $<$ Non-meditation) } \\
\hline Right isthmus cingulate & $-12.34(-20.73,-3.05)$ & $-2.00(-4.80,7.98)$ & $30(0.047,-0.50)$ \\
\hline Right pericalcarine & $-8.48(-11.20,-6.61)$ & $-0.85(-6.05,6.60)$ & $28(0.034,-0.53)$ \\
\hline Right posterior cingulate cortex & $-11.82(-18.97,2.93)$ & $11.49(0.56,15.20)$ & $29(0.040,-0.52)$ \\
\hline \multicolumn{4}{|c|}{ Gray Matter Volume (Meditation > Non-meditation) } \\
\hline Left caudal middle frontal & $4.47(-2.94,9.98)$ & $-6.38(-29.35,0.080)$ & $95(0.023,0.57)$ \\
\hline Left lateral occipital & $1.59(-7.93,5.66)$ & $-15.94(-24.76,-5.17)$ & $97(0.016,0.60)$ \\
\hline \multicolumn{4}{|l|}{ Gray Matter Volume (Meditat } \\
\hline Right entorhinal cortex & $-21.29(-38.83,-7.37)$ & $2.29(-13.87,19.97)$ & $27(0.028,-0.55)$ \\
\hline
\end{tabular}

The level of significance ( $p$-value), the rank biserial correlation effect size ( $r$-value) of the Mann-Whitney $U$ test is reported. 
significantly between meditation and non-meditation groups (Table 3). The results which meet the statistical criteria are discussed in this manuscript.

\section{Analysis of Structural Magnetic Resonance Images Data}

We compared cortical thickness and gray matter volume between meditation and non-meditation groups at baseline. We did not observe any significant differences between the groups for cortical thickness. We also did not find significant differences in gray matter volume, except in the left lateral occipital, right pars triangularis, and postcentral regions (Please refer to Supplementary Tables 1, 2).

Section "Regions of Interest-Based Analysis" and "VoxelWise Analysis" discuss the results of ROI-based and voxel-wise analysis, respectively.

\section{Regions of Interest-Based Analysis}

We did a comparative ROI-based analysis of cortical thickness and gray matter volume between meditation and non-meditation groups. Results highlighting regions showing significant changes between the meditation and non-meditation groups are shown in Table 4. In contrast with the non-meditation group, the meditation group showed significantly higher cortical thickness and gray matter volume in the left caudal and rostral middle frontal areas. The meditation group showed a significantly higher gray matter volume in left lateral occipital, right inferior parietal, and right superior frontal cortices. On the other hand, we also observed a significant decrease in cortical thickness and gray matter volume mainly in the entorhinal cortex and posterior parts of the brain.

\section{Voxel-Wise Analysis}

To further explore our data, we performed voxel-wise cortical thickness analysis (cortical thickness computed as the shortest distance between the gray/white matter junction and the pial surface), followed by a voxel-wise gray matter volume analysis. Table 5 and Figures 1A,B show the results of the cortical thickness analysis. Table $\mathbf{6}$ and Figures 2A,B show the results of the gray matter volume analysis.

For the visual inspection of increased or decreased cortical thickness and gray matter volume within each group, we displayed the mean SPC values in each group on inflated brain surfaces (see Figures 3, 4).

\section{Cortical Thickness}

Our results (Table 5 and Figures 1A,B, 3) showed significant effects of meditation mostly in the anterior parts of the brain, selectively in the frontal regions and in the occipitoparietal region in the posterior part. The meditation group showed significantly increased cortical thickness in the left superior parietal, left caudal middle frontal, left rostral middle frontal, left paracentral and in the right hemisphere, superior frontal, and lateral orbitofrontal regions. The meditation group also showed decreased thickness compared with the non-meditation group in the left lateral occipital, inferior parietal, inferior temporal, and superior temporal cortices, and on the right hemisphere in the lateral occipital, pericalcarine, postcentral,
TABLE 5 | Brain regions showing significant cortical thickness SPC changes (increase/decrease) in voxel-based analysis between the meditation and non-meditation groups corrected for multiple comparisons, age, gender, and education in both hemispheres.

\begin{tabular}{lcc}
\hline Brain region (TalX, TalY, TalZ) & Size $\left(\mathbf{m m}^{\mathbf{2}}\right)$ & $\boldsymbol{P}$-value \\
\hline Left Hemisphere & & \\
\hline SPC for Meditation $>$ Non-meditation & & \\
Superior parietal $(-24.1,-58.9,53.9)$ & 271.35 & $<0.00016$ \\
Rostral middle frontal $(-44.4,25,35.6)$ & 128.13 & $<0.00079$ \\
Caudal middle frontal $(-33.4,25,35.6)$ & 127.14 & $<0.00157$ \\
Paracentral $(-8.8,-22,55.7)$ & 55.79 & $<0.0047$ \\
Caudal middle frontal $(-34.1,7.2,55.6)$ & 53.52 & $<0.0030$ \\
SPC for Meditation $<$ Non-meditation & & \\
Lateral occipital $(-30,-83.6,-14.4)$ & 76.78 & $<0.00041$ \\
Inferior parietal $(-46.7,-58.4,10.4)$ & 73.47 & $<0.00053$ \\
Inferior temporal $(-45.5,-39.4,22)$ & 52.6 & $<0.00153$ \\
Superior temporal $(-54.8,-23.7,-3.5)$ & 52.19 & $<0.00226$ \\
\hline
\end{tabular}

Right Hemisphere

SPC for Meditation > Non-meditation

Superior frontal $(11.7,13.8,61.8)$

Lateral orbitofrontal $(23,20.3,-18.5)$

Superior frontal $(7.3,27.9,51.8)$

$<0.000067$

SPC for Meditation < Non-meditation

Lateral occipital $(24.7,-88,17.9)$

Pericalcarine $(21.8,-71,7.3)$

Postcentral (48.4, -23.4, 53.7)

Pericalcarine $(6.1,-87.2,7.5)$

Inferior parietal $(33.8,-71.2,35.5)$

Paracentral $(4.6,-26.3,68)$

Posterior cingulate $(13.4,-20,38)$

$\begin{aligned} 123.81 & <0.00029 \\ 51.91 & <0.0026 \\ 155.11 & <0.00054 \\ 132.54 & <0.00172 \\ 115.73 & <0.000028 \\ 104.21 & <0.00141 \\ 84.82 & <0.000035 \\ 69.77 & <0.00046 \\ 56.88 & <0.0047\end{aligned}$

TalX, $Y$, and $Z$ represent Talairach coordinates $X, Y$, and $Z$. Table includes only the regions which had cluster size greater than $50 \mathrm{~mm}^{2}$ and corresponding significance (p-value) less than 0.05 .

inferior parietal, paracentral, and posterior cingulate cortices. Changes in cortical thickness were observed asymmetrically over multiple regions either in the right or the left hemisphere, except in the inferior parietal and lateral occipital cortices where they were seen bilaterally.

\section{Gray Matter Volume}

In the whole-brain gray matter volume analysis (Table 6 and Figures $\mathbf{2 A}, \mathbf{B}, \mathbf{4}$ ), in contrast to the non-meditation group, the meditation group showed significantly higher gray matter volume bilaterally in the rostral middle frontal and superior frontal cortices. In the left hemisphere, the superior parietal and caudal middle frontal regions showed an increase in volume, as did the lateral orbitofrontal region in the right hemisphere. As shown in Figure 2A, the left superior frontal changes were observed mainly in the dorsomedial frontal regions. None of the brain regions showed significantly decreased gray matter volume in the meditation group compared to the non-meditation group.

\section{Volume Analysis of the Subcortical Structures}

On comparing the volume of subcortical structures including the cerebellum, thalamus, caudate, putamen, pallidum, and 


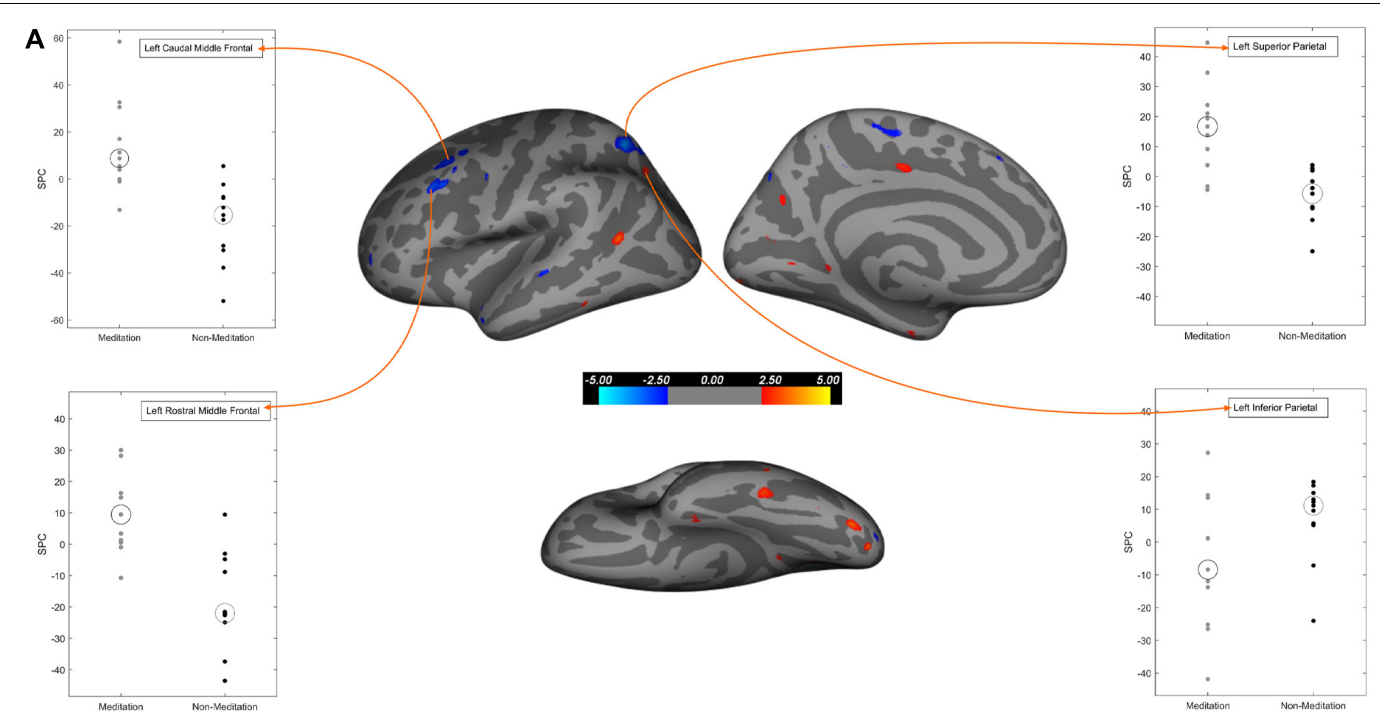

B

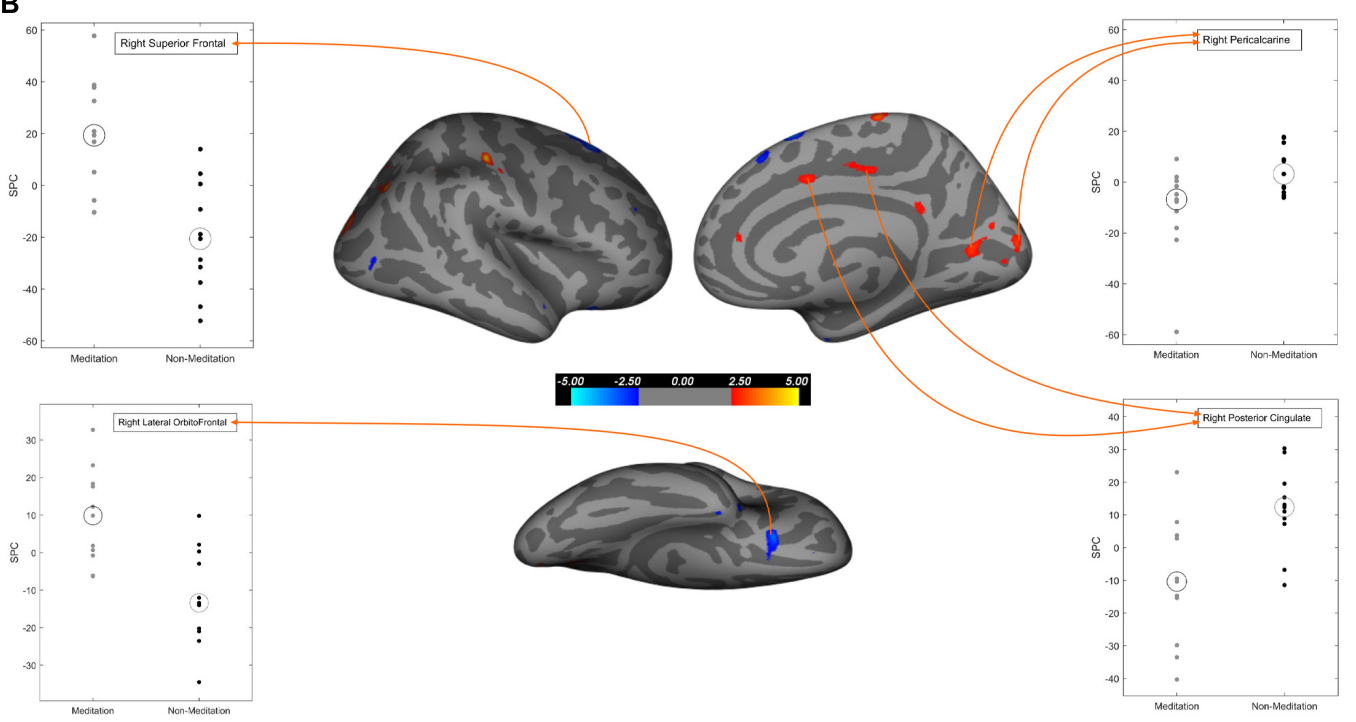

FIGURE 1 | Regions of cortical thickness that exhibit significant differences between meditation and non-meditation cohorts corrected for multiple comparisons, age, gender, and education. In all three different views (Lateral, medial, inferior) of brain surfaces, the blue clusters represent significantly higher SPC values while the red clusters represent significantly lesser SPC values in the meditation group compared to the non-meditation group. Graphs for significantly large cluster sizes are annotated on the inflated brain surface (see Table 5 for more details). (A) Left hemisphere; (B) Right hemisphere.

amygdala between the meditation and the non-meditation group, no significant changes in the left hemisphere were observed. However, we observed significantly increased volume in the right thalamus $(p<0.034)$ and a non-significant increasing trend in the right caudate $(p<0.056)$ in the meditation group.

\section{Hippocampal Subfields Analysis}

From the statistical analysis, we observed that the total hippocampal volume showed a positive rate of change in both the hemispheres from base to repeat scans in the meditation group, while in the non-meditation group, it decreased. Selectively, the volume of individual subfields in both hemispheres showed a positive change in the mediation group compared to the nonmediation group except in the fimbria (Figure 5). The left CA1 $(p<0.001)$, left molecular layer HP $(p<0.04)$, and left CA3 $(p<0.040)$ showed significantly higher SPC volume in the meditation group compared to the non-meditation group. The mean SPC values of each hippocampal subfield for the meditation and the non-meditation groups for both the hemispheres are plotted in Figure 5.

\section{DISCUSSION}

In this longitudinal study, we explored the effect of longterm daily meditation on the structural and neuropsychological changes in patients with MCI and mild AD. Our results show significant morphometric changes in cortical gray matter in the meditation group in a pattern that provides support to our hypotheses. 
TABLE 6 | Brain regions showing significant gray matter volume SPC changes (increase/decrease) in voxel-based analysis between the meditation and non-meditation groups corrected for multiple comparisons, age, gender, and education in both hemispheres.

\begin{tabular}{lcl}
\hline Brain region (TalX, TalY, TalZ) & Size $\left(\mathbf{m m}^{\mathbf{2}}\right)$ & $\boldsymbol{P}$-value \\
\hline Left Hemisphere & & \\
\hline SPC for Meditation $>$ Non-meditation & & \\
Lateral occipital $(-42.6,-75.9,4.5)$ & 281.47 & $<0.00021$ \\
Caudal middle frontal $(-32.4,13.3,47.6)$ & 225.47 & $<0.0025$ \\
Rostral middle frontal $(-44.1,26.9,28.1)$ & 136.1 & $<0.000073$ \\
Superior parietal $(-25.2,-57.3,51.6)$ & 127.19 & $<0.0010$ \\
Superior frontal $(-6.9,26.5,45.2)$ & 90.77 & $<0.0013$ \\
Banks of superior temporal sulcus $(-62,-34.3,4.6)$ & 87.22 & $<0.0027$ \\
Medial orbitofrontal $(-9.1,47.8,-8.8)$ & 64.64 & $<0.0020$ \\
Superior parietal $(-15.1,-68.2,47.2)$ & 53.04 & $<0.0011$ \\
Caudal middle frontal $(-32.2,23.8,42)$ & 48.32 & $<0.0040$
\end{tabular}

\section{Right Hemisphere}

\section{SPC for Meditation > Non-meditation}

Superior frontal $(18.6,14,60.2)$

Lateral orbitofrontal $(26.7,18.1,-20.5)$

Superior frontal $(10,-3.7,67)$

Rostral middle frontal $(44.7,31.8,21.6)$

Lateral orbitofrontal $(13.2,31.7,-17.8)$

$\begin{array}{ll}341.4 & <0.0002 \\ 70.23 & <0.0027 \\ 66.56 & <0.0030 \\ 62.13 & <0.0014 \\ 50.7 & <0.0005\end{array}$

TalX, Y, and $Z$ represent Talairach coordinates $X, Y$, and $Z$. Table includes only the regions which had cluster size greater than $50 \mathrm{~mm}^{2}$ and corresponding significance ( $p$-value) less than 0.05. None of the brain regions showed significantly decreased gray matter volume in the meditation group. The medial orbitofrontal region showed negative SPC values of gray matter volume for both groups but significantly less negative in the meditation group compared to the non-meditation group.

We used both ROI-based and voxel-wise analyses for our study. In within-group analysis between two-time points, we found a widespread increase in cortical thickness or gray matter volume in the meditation group, particularly prominently over the left prefrontal cortex, but also on the right. Conversely, we found reduced cortical thickness and gray matter volume following meditation in the posterior cingulate cortex, occipital cortex, and medial temporal regions, especially in the right hemisphere. Between-group comparison of meditators and nonmeditators using ROI data revealed that meditation significantly increased cortical thickness and gray matter volume in the caudal and rostral middle frontal cortices bilaterally and increased gray matter volume alone over the right superior frontal gyrus (SFG). Significantly reduced cortical thickness, gray matter volume, or both was seen over bilateral entorhinal and left parahippocampal cortices, right PCC and isthmus of cingulate, and right pericalcarine cortex.

A subsequent voxel-wise comparison over the prefrontal cortex showed significantly higher cortical thickness or gray matter volume in the left caudal middle frontal, bilateral rostral middle frontal, bilateral superior frontal, right lateral orbitofrontal, and left medial orbitofrontal cortices in the meditation group. The caudal middle frontal cortex is important for top-down modulation of attention such as in cognitive decision-making when confronted with competing visual stimuli or for re-orienting attention from stimulus-driven exogenous control to goal-directed endogenous control (Japee et al., 2015; Germann and Petrides, 2020). Cortical thinning in the caudal middle frontal region has been associated with poorer scores on attention and divided attention (Grambaite et al., 2011). It is therefore interesting that sustained meditation practice could strengthen the caudal middle frontal cortices in our patients. The rostral middle frontal cortex is important for attention, working memory, executive control, and emotion regulation (Miller and Cohen, 2001; Michalski et al., 2017) and are activated during response inhibition and switching performances (Grambaite et al., 2011). We speculate that the significantly increased cortical thickness and gray matter volume in the rostral middle frontal cortices in our patients may have followed repeated reinforcement of many of these functions by long-term meditation.

Previous works on healthy meditators have shown an increased cortical thickness or gray matter volume in the ventromedial orbitofrontal prefrontal cortex (Kang et al., 2013; Hernández et al., 2016). Unlike in healthy subjects, the orbitofrontal cortex might be thinner in patients with MCI and $\mathrm{AD}$ (Zhao et al., 2015). Our finding of a reversal of thinning of the orbitofrontal cortex in meditators is encouraging and suggests that long-term practice of meditation could reinforce the regions that control social cognition and behavior (Campos et al., 2019). The lateral orbitofrontal cortex has been associated with the evaluation and prediction of possible outcomes of an action. The medial orbitofrontal cortex takes this information and compares and contrasts the choices based on value, before selecting the appropriate action, possibly in conjunction with the dorsolateral prefrontal cortex (Rudebeck and Murray, 2014). One of the distinctive features of meditation on breath or on witnessing thoughts is the default actions of mind-wandering or rise and fall of thoughts, respectively. The mind repeatedly gravitates out of attention and requires constant updating, the evaluation of choices, and value-based selection to bring it back to the task at hand. Although we are not aware of specific physiological studies on the role of the orbitofrontal cortex in meditation, it is conceivable how meditation could potentially help reinforce the functions of the lateral and medial orbitofrontal cortices.

The superior frontal gyrus (SFG) has been associated with working memory (du Boisgueheneuc et al., 2006; Nissim et al., 2017), cognitive control, cognitive execution, and motor control (Li et al., 2013) and spatial cognition orientation (du Boisgueheneuc et al., 2006). Lack of cognitive control by the SFG has been implicated in impulsivity (Hu et al., 2016) and poor social functioning (Tully et al., 2014). The increased cortical thickness or gray matter volume in our subjects could be because of the sustained control achieved by meditation over externally or internally arising distractions.

The meditation group showed significantly increased cortical thickness of the left superior parietal lobule and decreased cortical thickness of bilateral inferior parietal lobules. The superior parietal lobule, the intraparietal sulcus, and the frontal eye field form parts of the dorsal attentional network that controls topdown, goal-oriented visual attention (Corbetta and Shulman, 1998; Kastner et al., 1999). The right inferior parietal lobule 

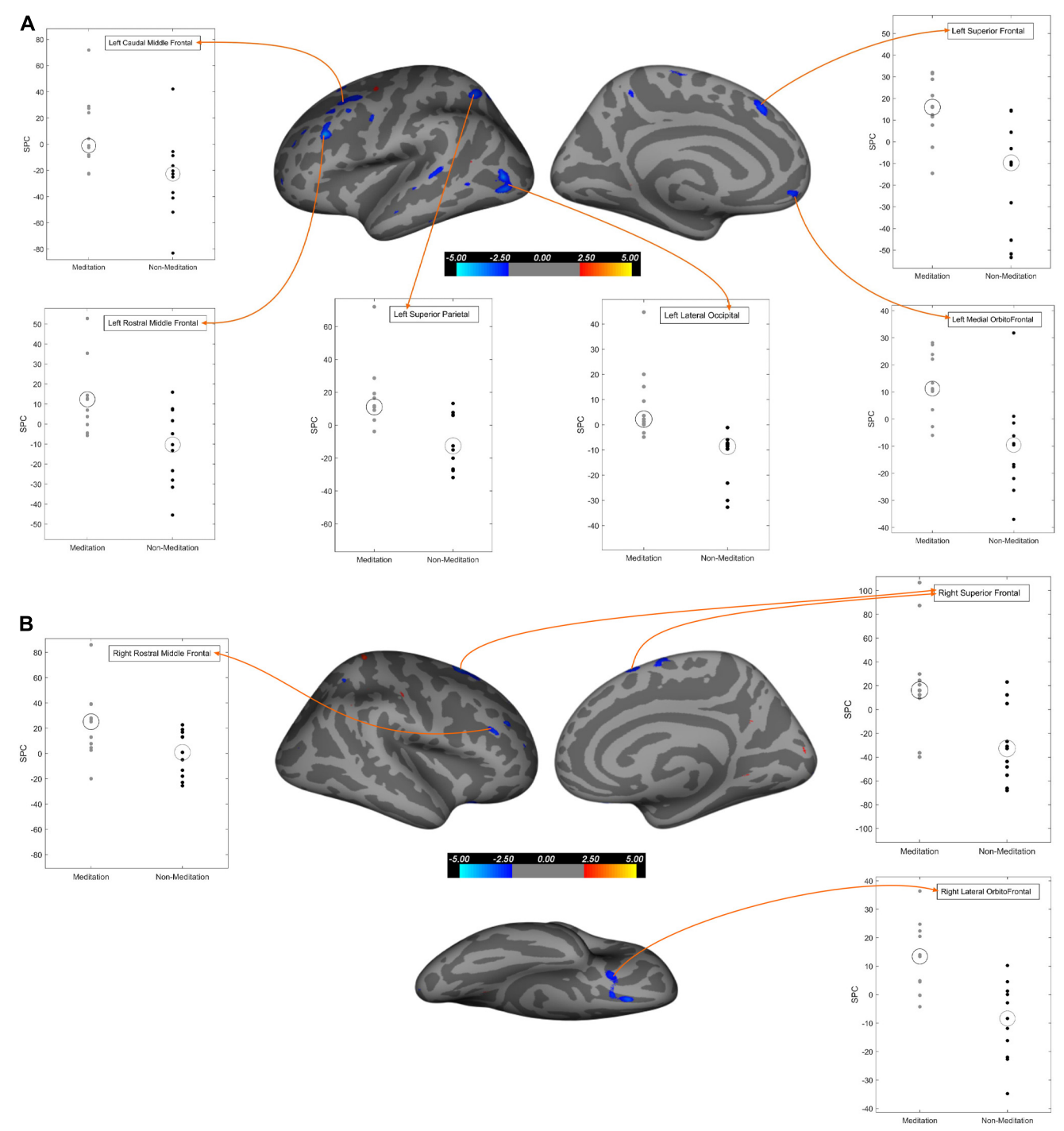

FIGURE 2 | Regions of gray matter volume that exhibit significant differences between meditation and non-meditation cohorts corrected for multiple comparisons, age, gender, and education. In all three different views (Lateral, medial, inferior) of brain surfaces, the blue clusters represent significantly higher SPC values while the red clusters represent significantly lesser SPC values in the meditation group compared to the non-meditation group. Graphs for significantly large cluster sizes are annotated on the inflated brain surface (see Table 6 for more details). (A) Left hemisphere; (B) Right hemisphere.

(including the temporoparietal junction) is part of the ventral attentional network that mediates bottom-up responses driven by salient or unexpected stimuli (Corbetta et al., 2008). The two networks appear to be controlled by a "circuit breaker," allowing the required flexibility and balance between attending to the goaloriented responses and stimulus-driven, potentially distracting responses (Corbetta and Shulman, 2002; Behrmann et al., 2004; Fox et al., 2006; Shomstein, 2012; Japee et al., 2015). Meditation, therefore, appears to have strengthened regions subserving topdown control, while attenuating those that oversee stimulusdriven responses.

Significantly more thinning of the right PCC in our meditation group parallels similar findings by others, albeit using a different meditation technique (Kang et al., 2013). The PCC is central to the functioning of the DMN and is active during mind wandering, daydreaming, planning for the future, and retrieval of autobiographical memory. Modulation of the PCC activity is important for effectively switching from a broad, unrestrained attention to the more sharply focused attention to a salient external stimulus (Sridharan et al., 2008; Menon and Uddin, 2010; Leech and Sharp, 2014). The PCC and the hippocampus lose structural and functional integrity early in the course of AD (Greicius et al., 2004) thereby not only affecting autobiographical and episodic memory but also weakening the anticorrelation of the DMN with the frontoparietal attentional networks. In addition to the right PCC, significantly more cortical thinning or shrinkage of gray matter volume in meditators was also seen in the left parahippocampal cortex and the entorhinal cortices bilaterally, along with similar changes in the inferior parietal cortices, mentioned earlier. It is conceivable that meditation, by 

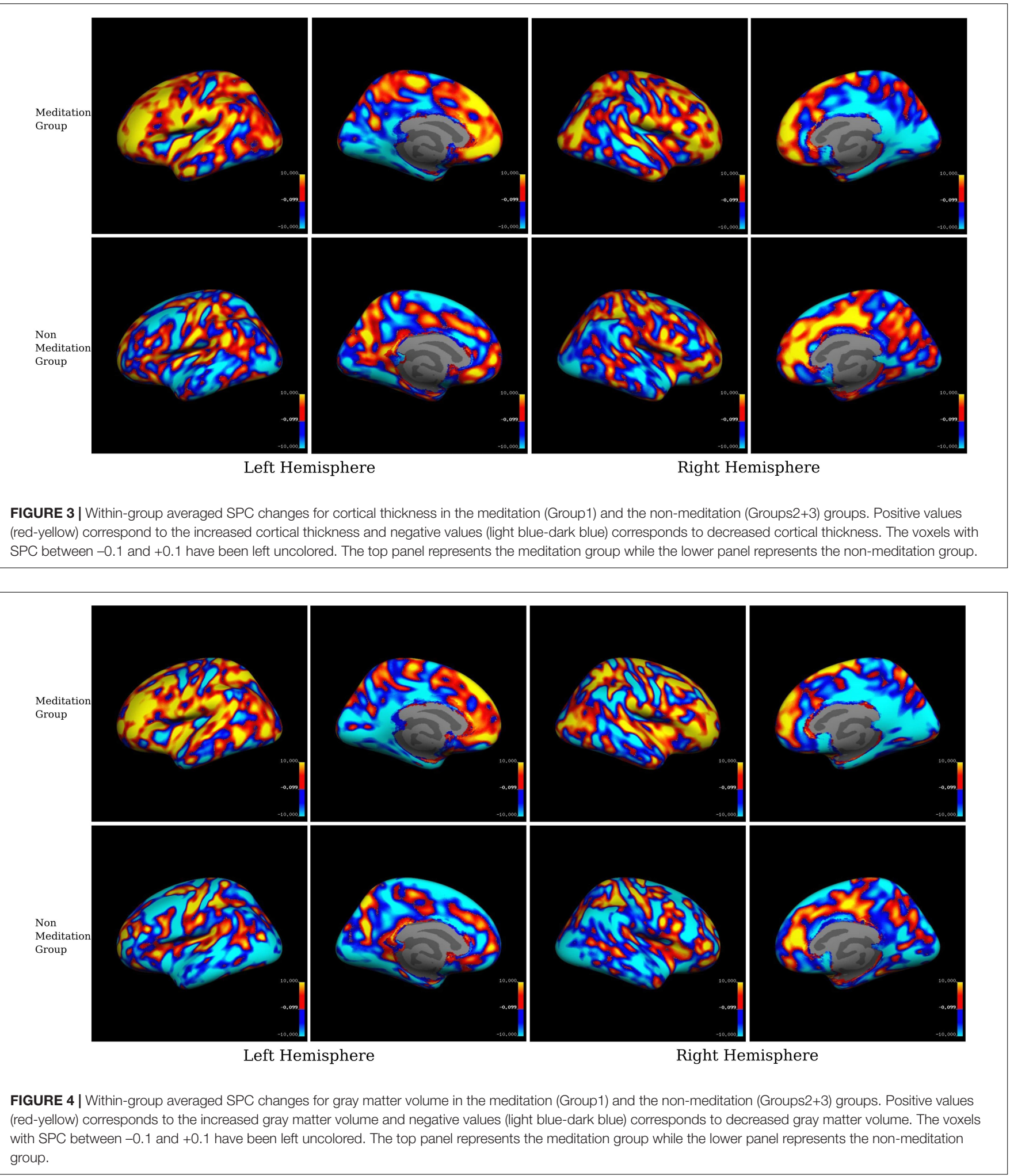

enhancing attentional and executive control and by reducing mind wandering, further shifts neural activity away from the already degenerated posterior DMN structures to the prefrontal cortex. However, the hippocampal substructures themselves tended to show a greater increase in volume in the meditation group, and significant increases were seen in the left CA1, CA3, and the molecular layer when compared with the non-meditation group. This seems counterintuitive and difficult to explain easily 

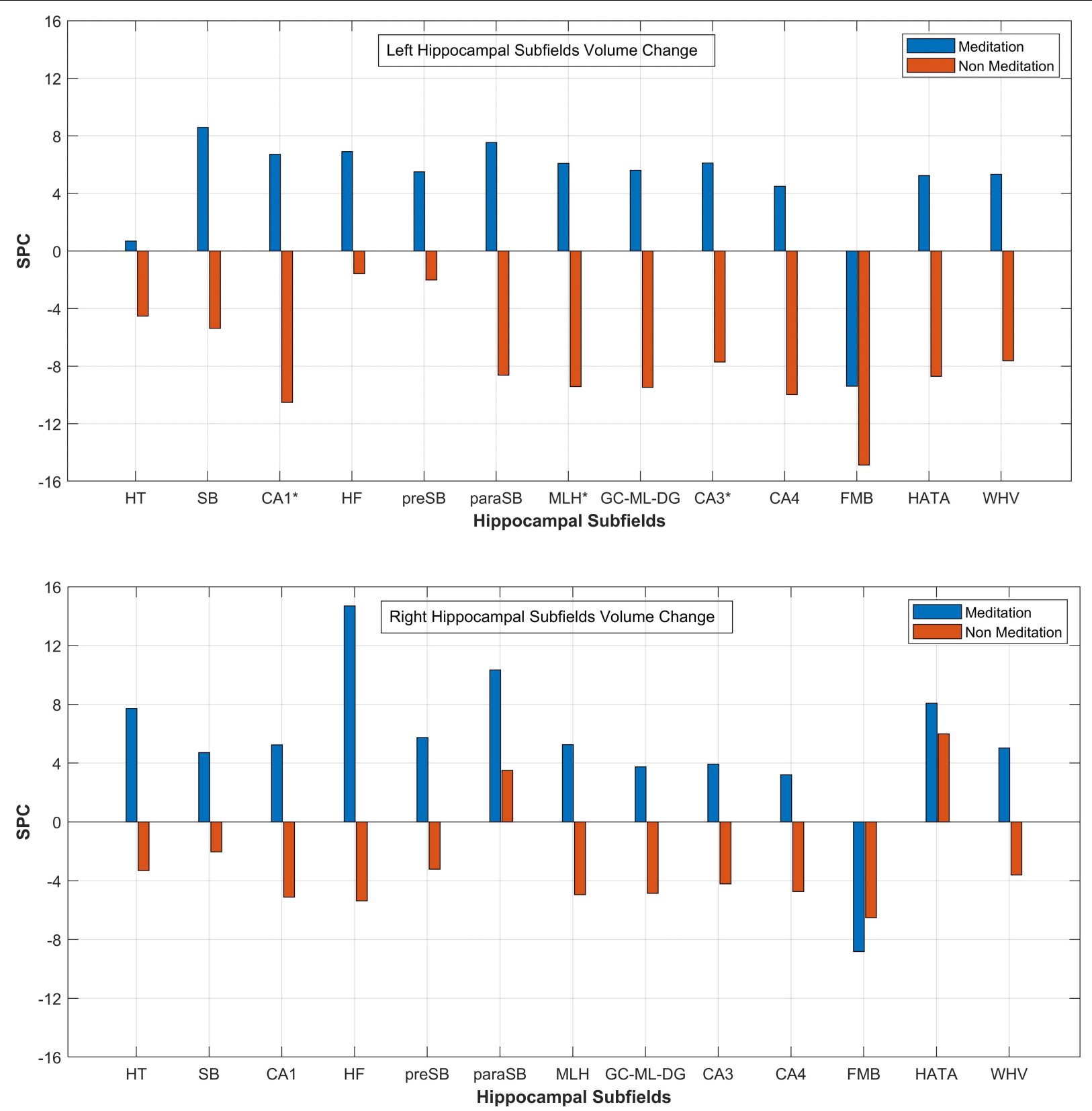

FIGURE 5 | Mean SPC values for volume of each Left and Right Hippocampal Subfields respectively for the mediation and the non-meditation groups. HT, hippocampal tail; SB, subiculum; CA1, HF, hippocampal fissure; preSB, presubiculum; paraSB, parasubiculum; MLH, molecular layer HP; GC-ML-DG, [Granule Cell (GC) and Molecular Layer (ML) of the Dentate Gyrus (DG)]; FMB, fimbria; HATA (hippocampus-amygdala-transition-area); WHV, Whole Hippocampal Volume. ${ }^{*}$ Corresponds to significant changes in the subfield's volume.

with our current data. Nevertheless, the effect of meditation in slowing down hippocampal volume loss in MCI patients has been described earlier (Wells et al., 2013b).

Our finding of lesser cortical thickness in bilateral lateral occipital cortices, right pericalcarine cortex, as well as the postcentral cortex in the meditating group is consistent with the anterior-posterior gradient seen in meditation, with more cortical thinning in posterior brain regions (Kang et al., 2013). Given that occipital thinning in these regions has been reported in
MCI and AD (Long et al., 2018; Yang et al., 2019; Edmonds et al., 2020), it is possible that our meditation techniques, by reinforcing the anterior predominant regions responsible for topdown control and suppressing the posterior bottom-up stimulusdriven regions, further promoted the most optimal use of limited cognitive resources.

It would be interesting to consider whether the relatively less thinning in the posterior brain regions in non-meditators could have partly resulted from the positive effect on these structures 
in the coloring task group. Indeed, the right posterior cingulate cortex was thicker in non-meditators at follow-up. Although we are not aware of previous morphometric studies in this context, functional connectivity studies have shown increased activation of the PCC/precuneus and its frontal and parietal connections following visual art production (Bolwerk et al., 2014). However, the subjects engaged in our coloring task were too few and any further speculation can only be done after a larger study. Moreover, even though both meditation and the coloring tasks require attention and top-down control, meditation, by actively discouraging mind wandering, may lead to increased suppression of the posterior brain regions and consequent loss of gray matter, as seen in our study and by others (Kang et al., 2013).

Among the subcortical structures, only the right thalamus showed a significant volume increase among the meditators. This is in keeping with previous reports on the positive effects of meditation on the thalamus (Luders et al., 2009; Newberg et al., 2010; Laneri et al., 2016). A possible explanation for this is the heightened sensory awareness during meditation (Laneri et al., 2016). This allows the meditator to observe a continual flow of sensory stimuli that is a part of the meditation experience.

Contrary to the positive structural changes in the brain, we could not demonstrate significant cognitive improvement following meditation. This is despite increases in gray matter in areas known to control attention, executive function, and memory. Nevertheless, we observed a trend toward improved attention in meditators in the digit forward task. In a recent study (Yu et al., 2021), significantly improved attention, and better performance in color trails interference task were seen after 9 months of mindfulness meditation in MCI subjects, but the effects were not seen after correcting for false discovery rate. No other neuropsychological function changed significantly in that study during this period (Yu et al., 2021). Indeed, results from the few studies that have looked at the cognitive effects of meditation in MCI or AD patients have been variable, with some showing no significant improvement compared to controls (Wells et al., 2013a; Larouche et al., 2018, 2019; Klainin-Yobas et al., 2019). It is possible that a longer follow-up would bring out the cognitive benefits, as has been noted in some of the studies (QuintanaHernández et al., 2016; Wong et al., 2017). Alternatively, the standard neuropsychological tests being used may not have been appropriate to pick up early cognitive changes in these subjects.

To ensure robust and meaningful morphometric analysis with a limited sample size, we chose to report only those regions showing significant difference between the meditation and nonmeditation groups and having a minimum cluster size of over $50 \mathrm{~mm}^{2}$. The large effect sizes of more than 0.5 in all such regions in the ROI based analysis is comparable to the effect sizes reported by others (Yang et al., 2016; Yu et al., 2021).

\section{Limitations of the Study}

The high attrition due to the pandemic in our study meant that we had to work with a smaller sample than planned and had to club the control groups together for our analysis. It is possible that our results could have been different with a bigger sample. Although we cannot rule out some attrition bias contributing to our results, the dropouts were across the groups, the majority occurred early in the study, and the later ones were uniformly out of fear of the pandemic. All dropouts, either from neuropsychology tests or imaging, were excluded from the respective data analysis. Also, the final sample retained the originally planned ratio among the groups.

We recognize that an objective measure of the degree of training achieved by each participant would have been useful. However, our training sessions were on a one-to-one basis, and at the end of each training session, we did ask the subject to discuss his/her performance for the day, and every effort was made by the trainer to clear all doubts. A home-based longitudinal study must rely on self-reporting and carries a reporting bias. Our patients were encouraged to keep a log of their practice, but few maintained this regularly. However, we maintained regular telephonic contact with the patients in the meditation group and the active control group, and with their caregivers, to check for compliance and to address queries related to the task. In addition, patients in the active control group were required to submit their coloring books before collecting the next one.

For the hippocampal subfields analysis, we have used isotropic $1 \mathrm{~mm}^{3} \mathrm{~T} 1-\mathrm{w}$ images using automatic segmentation of Freesurfer 6.0. It is possible that higher resolution images could have provided more accurate results (Wisse et al., 2021).

We also acknowledge that the results of a non-randomized study with consecutive sampling are subject to selection bias. Our observations will need to be confirmed by future large randomized-control studies designed to address these questions.

\section{CONCLUSION}

In this study, we investigated the effects of long-term meditation on structural brain imaging parameters of MCI and mild AD patients using surface-based morphometric analysis. An increase in gray matter predominantly over the prefrontal cortex and a decrease more posteriorly suggest a shift toward increasing topdown control in the meditators. More importantly, it suggests that long-term daily meditation may be able to slow-down the neurodegenerative process in crucial areas of the brain. The lack of objective benefit in neuropsychological tests is not necessarily disheartening and may become evident after longer periods of observation, or earlier by using person-centered tests that can evaluate cognition in day-to-day tasks. Unlike previous studies on the subject, we employed a silent, sitting meditation technique that excluded mantras, yogic exercises, or body movements. We could therefore include a longer meditation routine than those used by some other researchers. To our knowledge, this is also the first study on morphometric changes caused by meditation to include patients with $\mathrm{AD}$. Our results need to be validated on a larger cohort observed at more time points. Investigation of other imaging modalities to analyze structural changes, especially the fiber pathways connecting different regions as well as their relation to the functional connectivity changes would offer us a more comprehensive account of the longitudinal effects of meditation intervention in neurodegenerative diseases. 


\section{DATA AVAILABILITY STATEMENT}

The original contributions presented in the study are included in the article/Supplementary Material, further inquiries can be directed to the corresponding author/s.

\section{ETHICS STATEMENT}

The studies involving human participants were reviewed and approved by Ethics Committee, Apollo Gleneagles Hospital, Kolkata, India. The patients/participants provided their written informed consent to participate in this study.

\section{AUTHOR CONTRIBUTIONS}

AG and ND conceptualized the study and arranged for funding. $A G$ and RSB supervised the study. AG, ND, JB, RB, and MG contributed to the study design. $\mathrm{AG}, \mathrm{ND}, \mathrm{MeD}$, and GP acquired the data. $\mathrm{MaD}, \mathrm{AP}, \mathrm{ND}, \mathrm{AG}$, and $\mathrm{RSB}$ contributed to data analysis and interpretation. $\mathrm{MaD}, \mathrm{AP}, \mathrm{ND}$, and $\mathrm{AG}$ drafted the manuscript. The manuscript was revised by all authors and approved the submitted version.

\section{REFERENCES}

Albert, M. S., DeKosky, S. T., Dickson, D., Dubois, B., Feldman, H. H., Fox, N. C., et al. (2011). The diagnosis of mild cognitive impairment due to Alzheimer's disease: recommendations from the National Institute on Aging-Alzheimer's Association workgroups on diagnostic guidelines for Alzheimer's disease. Alzheimers Dement. 7, 270-279. doi: 10.1016/j.jalz.2011. 03.008

Banerjee, R., and Chatterjee, A. (2018). Indian Philosophy and Meditation: Perspectives on Consciousness. London: Routledge, 164.

Behrmann, M., Geng, J. J., and Shomstein, S. (2004). Parietal cortex and attention. Curr. Opin. Neurobiol. 14, 212-217. doi: 10.1016/j.conb.2004. 03.012

Benjamini, Y., and Hochberg, Y. (1995). Controlling the false discovery rate: a practical and powerful approach to multiple testing. J. R. Stat. Soc. Ser. B Methodol. 57, 289-300.

Bolwerk, A., Mack-Andrick, J., Lang, F. R., Dörfler, A., and Maihöfner, C. (2014). How art changes your brain: differential effects of visual art production and cognitive art evaluation on functional brain connectivity. PLoS One 9:e101035. doi: 10.1371/journal.pone.0101035

Buddhaghosa, B., and Nanamoli, B. (2003). Path of Purification: Visuddhimagga. Onalaska, WA: BPS Pariyatti Editions, 950.

Campos, D., Modrego-Alarcón, M., López-del-Hoyo, Y., González-Panzano, M., Van Gordon, W., Shonin, E., et al. (2019). Exploring the role of meditation and dispositional mindfulness on social cognition domains: a controlled study. Front. Psychol. 10:809. doi: 10.3389/fpsyg.2019. 00809

Chételat, G., Mézenge, F., Tomadesso, C., Landeau, B., Arenaza-Urquijo, E., Rauchs, G., et al. (2017). Reduced age-associated brain changes in expert meditators: a multimodal neuroimaging pilot study. Sci. Rep. 7:10160. doi: 10.1038/s41598-017-07764-x

Corbetta, M., and Shulman, G. L. (1998). Human cortical mechanisms of visual attention during orienting and search. Philos. Trans. R. Soc. Lond. B Biol. Sci. 353, 1353-1362. doi: 10.1098/rstb.1998.0289

Corbetta, M., and Shulman, G. L. (2002). Control of goal-directed and stimulusdriven attention in the brain. Nat. Rev. Neurosci. 3, 201-215. doi: 10.1038/ $\operatorname{nrn} 755$

\section{FUNDING}

This study was funded through a research grant to AG by the Department of Science and Technology (DST) research program called "Science and Technology of Yoga and Meditation" (SATYAM) under its Cognitive Science Research Initiative (CSRI), India [Grant no. DST/SATYAM/2017/184(G)].

\section{ACKNOWLEDGMENTS}

The authors would like to thank JR Ram for allowing us to examine his patients. The authors would also like to thank Devyani Todi, Purbasha Mukherjee, and Anish Kar for helping us with data compilation.

\section{SUPPLEMENTARY MATERIAL}

The Supplementary Material for this article can be found online at: https://www.frontiersin.org/articles/10.3389/fnhum. 2021.728993/full\#supplementary-material

Corbetta, M., Patel, G., and Shulman, G. L. (2008). The reorienting system of the human brain: from environment to theory of mind. Neuron 58, 306-324. doi: 10.1016/j.neuron.2008.04.017

Desikan, R. S., Ségonne, F., Fischl, B., Quinn, B. T., Dickerson, B. C., Blacker, D., et al. (2006). An automated labeling system for subdividing the human cerebral cortex on MRI scans into gyral based regions of interest. NeuroImage 31, 968-980. doi: 10.1016/j.neuroimage.2006.01.021

Dipasquale, O., and Cercignani, M. (2016). Network functional connectivity and whole-brain functional connectomics to investigate cognitive decline in neurodegenerative conditions. Funct. Neurol. 31, 191-203. doi: 10.11138/fneur/ 2016.31.4.191

du Boisgueheneuc, F., Levy, R., Volle, E., Seassau, M., Duffau, H., Kinkingnehun, S., et al. (2006). Functions of the left superior frontal gyrus in humans: a lesion study. Brain 129, 3315-3328. doi: 10.1093/brain/awl244

Du, A.-T., Schuff, N., Kramer, J. H., Rosen, H. J., Gorno-Tempini, M. L., Rankin, K., et al. (2007). Different regional patterns of cortical thinning in Alzheimer's disease and frontotemporal dementia. Brain 130, 1159-1166. doi: 10.1093/ brain/awm016

Edmonds, E. C., Weigand, A. J., Hatton, S. N., Marshall, A. J., Thomas, K. R., Ayala, D. A., et al. (2020). Patterns of longitudinal cortical atrophy over 3 years in empirically derived MCI subtypes. Neurology 94, e2532-e2544. doi: 10.1212/WNL.0000000000009462

Fam, J., Sun, Y., Qi, P., Lau, R. C., Feng, L., Kua, E. H., et al. (2020). Mindfulness practice alters brain connectivity in community-living elders with mild cognitive impairment. Psychiatry Clin. Neurosci. 74, 257-262. doi: 10.1111/ pcn.12972

Fazekas, F., Chawluk, J. B., Alavi, A., Hurtig, H. I., and Zimmerman, R. A. (1987). MR signal abnormalities at $1.5 \mathrm{~T}$ in Alzheimer's dementia and normal aging. AJR Am. J. Roentgenol. 149, 351-356. doi: 10.2214/ajr.149.2.351

Fischl, B., Liu, A., and Dale, A. M. (2001). Automated manifold surgery: constructing geometrically accurate and topologically correct models of the human cerebral cortex. IEEE Trans. Med. Imaging 20, 70-80. doi: 10.1109/42. 906426

Fox, K. C. R., Dixon, M. L., Nijeboer, S., Girn, M., Floman, J. L., Lifshitz, M., et al. (2016). Functional neuroanatomy of meditation: a review and metaanalysis of 78 functional neuroimaging investigations. Neurosci. Biobehav. Rev. 65, 208-228. doi: 10.1016/j.neubiorev.2016.03.021 
Fox, K. C. R., Nijeboer, S., Dixon, M. L., Floman, J. L., Ellamil, M., Rumak, S. P., et al. (2014). Is meditation associated with altered brain structure? A systematic review and meta-analysis of morphometric neuroimaging in meditation practitioners. Neurosci. Biobehav. Rev. 43, 48-73. doi: 10.1016/j. neubiorev.2014.03.016

Fox, M. D., Corbetta, M., Snyder, A. Z., Vincent, J. L., and Raichle, M. E. (2006). Spontaneous neuronal activity distinguishes human dorsal and ventral attention systems. Proc. Natl. Acad. Sci. U.S.A. 103, 10046-10051.

Gauthier, S., Reisberg, B., Zaudig, M., Petersen, R. C., Ritchie, K., Broich, K., et al. (2006). Mild cognitive impairment. Lancet 367, 1262-1270. doi: 10.1016/S01406736(06)68542-5

Germann, J., and Petrides, M. (2020). Area 8A within the posterior middle frontal gyrus underlies cognitive selection between competing visual targets. eNeuro 7 , 1-12. doi: 10.1523/ENEURO.0102-20.2020

Grambaite, R., Selnes, P., Reinvang, I., Aarsland, D., Hessen, E., Gjerstad, L., et al. (2011). Executive dysfunction in mild cognitive impairment is associated with changes in frontal and cingulate white matter tracts. J. Alzheimers Dis.? 27, 453-462. doi: 10.3233/JAD-2011-110290

Greicius, M. D., Srivastava, G., Reiss, A. L., and Menon, V. (2004). Defaultmode network activity distinguishes Alzheimer's disease from healthy aging: evidence from functional MRI. Proc. Natl. Acad. Sci. U.S.A. 101, 4637-4642. doi: 10.1073/pnas.0308627101

Hasenkamp, W., and Barsalou, L. W. (2012). Effects of meditation experience on functional connectivity of distributed brain networks. Front. Hum. Neurosci. 6:38. doi: $10.3389 /$ fnhum. 2012.00038

Hernández, S. E., Suero, J., Barros, A., González-Mora, J. L., and Rubia, K. (2016). Increased grey matter associated with long-term Sahaja yoga meditation: a voxel-based morphometry study. PLoS One 11:e0150757. doi: 10.1371/journal. pone. 0150757

Hu, S., Ide, J. S., Zhang, S., and Li, C.-S. R. (2016). The right superior frontal gyrus and individual variation in proactive control of impulsive response. J. Neurosci. 36, 12688-12696. doi: 10.1523/JNEUROSCI.1175-16.2016

Jack, C. R., Albert, M. S., Knopman, D. S., McKhann, G. M., Sperling, R. A., Carrillo, M. C., et al. (2011). Introduction to the recommendations from the National Institute on Aging-Alzheimer's Association workgroups on diagnostic guidelines for Alzheimer's disease. Alzheimers Dement. 7, 257-262. doi: 10. 1016/j.jalz.2011.03.004

Jack, C. R., Bennett, D. A., Blennow, K., Carrillo, M. C., Dunn, B., Haeberlein, S. B., et al. (2018). NIA-AA research framework: toward a biological definition of Alzheimer's disease. Alzheimers Dement. 14, 535-562. doi: 10.1016/j.jalz.2018. 02.018

Japee, S., Holiday, K., Satyshur, M. D., Mukai, I., and Ungerleider, L. G. (2015). A role of right middle frontal gyrus in reorienting of attention: a case study. Front. Syst. Neurosci. 9:23. doi: 10.3389/fnsys.2015.00023

JASP Team (2020). JASP (0.14.1)[Computer software].

Kabat-Zinn, J. (1990). Full Catastrophe Living: Using the Wisdom of Your Body and Mind to Face Stress, Pain, and Illness. New York, NY: Delacorte Press, doi: $10.1037 / 032287$

Kang, D.-H., Jo, H. J., Jung, W. H., Kim, S. H., Jung, Y.-H., Choi, C.-H., et al. (2013). The effect of meditation on brain structure: cortical thickness mapping and diffusion tensor imaging. Soc. Cogn. Affect. Neurosci. 8, 27-33. doi: 10.1093/ scan/nss056

Kastner, S., Pinsk, M. A., De Weerd, P., Desimone, R., and Ungerleider, L. G. (1999). Increased activity in human visual cortex during directed attention in the absence of visual stimulation. Neuron 22, 751-761. doi: 10.1016/S08966273(00)80734-5

Kim, H. J., Jae Baek, M., and Kim, S. Y. (2014). Alternative type of the trail making test in nonnative english-speakers: the trail making test-black \& white. PLoS One 9:e89078. doi: 10.1371/journal.pone.0089078

Klainin-Yobas, P., Kowitlawakul, Y., Lopez, V., Tang, C. T., Hoek, K. E., Gan, G. L., et al. (2019). The effects of mindfulness and health education programs on the emotional state and cognitive function of elderly individuals with mild cognitive impairment: a randomized controlled trial. J. Clin. Neurosci. 68, 211-217. doi: 10.1016/j.jocn.2019.05.031

Laneri, D., Schuster, V., Dietsche, B., Jansen, A., Ott, U., and Sommer, J. (2016). Effects of long-term mindfulness meditation on brain's white matter microstructure and its aging. Front. Aging Neurosci. 7:254. doi: 10.3389/fnagi. 2015.00254
Larouche, E., Hudon, C., and Goulet, S. (2018). Investigating a mindfulness-based intervention as an attentional network training to improve cognition in older adults with amnestic mild cognitive impairment: a randomized-controlled trial. Neuropsychol. Clin. Appl. 2, 110-125. doi: 10.46278/j.ncacn.20180505

Larouche, E., Hudon, C., and Goulet, S. (2019). Mindfulness mechanisms and psychological effects for aMCI patients: a comparison with psychoeducation. Complement Ther. Clin. Pract. 34, 93-104. doi: 10.1016/j.ctcp.2018.11.008

Leech, R., and Sharp, D. J. (2014). The role of the posterior cingulate cortex in cognition and disease. Brain 137, 12-32. doi: 10.1093/brain/awt162

Li, W., Qin, W., Liu, H., Fan, L., Wang, J., Jiang, T., et al. (2013). Subregions of the human superior frontal gyrus and their connections. NeuroImage 78, 46-58. doi: 10.1016/j.neuroimage.2013.04.011

Long, X., Jiang, C., and Zhang, L. (2018). Morphological biomarker differentiating MCI converters from nonconverters: longitudinal evidence based on hemispheric asymmetry. Behav. Neurol. 2018:3954101. doi: 10.1155/2018/3954101

Luders, E., Cherbuin, N., and Kurth, F. (2015). Forever Young(er): potential agedefying effects of long-term meditation on gray matter atrophy. Front. Psychol. 5:1551. doi: 10.3389/fpsyg.2014.01551

Luders, E., Toga, A. W., Lepore, N., and Gaser, C. (2009). The underlying anatomical correlates of long-term meditation: larger hippocampal and frontal volumes of gray matter. Neuroimage 45, 672-678.

Lutz, A., Slagter, H. A., Dunne, J. D., and Davidson, R. J. (2008). Attention regulation and monitoring in meditation. Trends Cogn. Sci. 12, 163-169. doi: 10.1016/j.tics.2008.01.005

Menon, V., and Uddin, L. Q. (2010). Saliency, switching, attention and control: a network model of insula function. Brain Struct. Funct. 214, 655-667. doi: 10.1007/s00429-010-0262-0

Michalski, L. J., Demers, C. H., Baranger, D. A. A., Barch, D. M., Harms, M. P., Burgess, G. C., et al. (2017). Perceived stress is associated with increased rostral middle frontal gyrus cortical thickness: a family-based and discordant-sibling investigation. Genes Brain Behav. 16, 781-789. doi: 10.1111/gbb.12404

Miller, E. K., and Cohen, J. D. (2001). An integrative theory of prefrontal cortex function. Annu. Rev. Neurosci. 24, 167-202. doi: 10.1146/annurev.neuro.24.1. 167

Morris, J. C. (1993). The clinical dementia rating (CDR): current version and scoring rules. Neurology 43, 2412-2412. doi: 10.1212/WNL.43.11.2412-a

Morris, J. C., Heyman, A., Mohs, R. C., Hughes, J. P., van Belle, G., Fillenbaum, G., et al. (1989). The consortium to establish a registry for Alzheimer's disease (CERAD). Part I. Clinical and neuropsychological assessment of Alzheimer's disease. Neurology 39, 1159-1165. doi: 10.1212/wnl.39.9.1159

Nasreddine, Z. S., Phillips, N. A., Bédirian, V., Charbonneau, S., Whitehead, V., Collin, I., et al. (2005). The Montreal cognitive assessment, MoCA: a brief screening tool for mild cognitive impairment. J. Am. Geriatr. Soc. 53, 695-699. doi: $10.1111 / j .1532-5415.2005 .53221 . x$

Newberg, A. B., Wintering, N., Khalsa, D. S., Roggenkamp, H., and Waldman, M. R. (2010). Meditation Effects on cognitive function and cerebral blood flow in subjects with memory loss: a preliminary study. J. Alzheimers Dis. 20, 517-526. doi: 10.3233/JAD-2010-1391

Nissim, N. R., O'Shea, A. M., Bryant, V., Porges, E. C., Cohen, R., and Woods, A. J. (2017). Frontal structural neural correlates of working memory performance in older adults. Front. Aging Neurosci. 8:328. doi: 10.3389/fnagi.2016.00328

O’Bryant, S. E., Waring, S. C., Cullum, C. M., Hall, J., Lacritz, L., Massman, P. J., et al. (2008). Staging dementia using clinical dementia rating scale sum of boxes scores: a Texas Alzheimer's research consortium study. Arch. Neurol. 65, 1091-1095. doi: 10.1001/archneur.65.8.1091

Pegueroles, J., Vilaplana, E., Montal, V., Sampedro, F., Alcolea, D., CarmonaIragui, M., et al. (2017). Longitudinal brain structural changes in preclinical Alzheimer's disease. Alzheimers Dement. 13, 499-509. doi: 10.1016/j.jalz.2016. 08.010

Petersen, R. C., Doody, R., Kurz, A., Mohs, R. C., Morris, J. C., Rabins, P. V., et al. (2001). Current concepts in mild cognitive impairment. Arch. Neurol. 58, 1985-1992. doi: 10.1001/archneur.58.12.1985

Quintana-Hernández, D. J., Miró-Barrachina, M. T., Ibáñez-Fernández, I. J., Pino, A. S.-D., Quintana-Montesdeoca, M. P., Rodríguez-de Vera, B., et al. (2016). Mindfulness in the maintenance of cognitive capacities in Alzheimers disease: a randomized clinical trial. J. Alzheimers Dis. 50, 217-232. doi: 10.3233/JAD143009 
Reuter, M., Schmansky, N. J., Rosas, H. D., and Fischl, B. (2012). Within-subject template estimation for unbiased longitudinal image analysis. NeuroImage 61, 1402-1418. doi: 10.1016/j.neuroimage.2012.02.084

Rudebeck, P. H., and Murray, E. A. (2014). The orbitofrontal oracle: cortical mechanisms for the prediction and evaluation of specific behavioral outcomes. Neuron 84, 1143-1156. doi: 10.1016/j.neuron.2014.10.049

Seeley, W. W., Menon, V., Schatzberg, A. F., Keller, J., Glover, G. H., Kenna, H., et al. (2007). Dissociable intrinsic connectivity networks for salience processing and executive control. J. Neurosci. 27, 2349-2356. doi: 10.1523/JNEUROSCI. 5587-06.2007

Ségonne, F., Pacheco, J., and Fischl, B. (2007). Geometrically accurate topologycorrection of cortical surfaces using nonseparating loops. IEEE Trans. Med. Imaging 26, 518-529. doi: 10.1109/TMI.2006.887364

Shomstein, S. (2012). Cognitive functions of the posterior parietal cortex: topdown and bottom-up attentional control. Front. Integr. Neurosci. 6:38. doi: $10.3389 /$ fnint.2012.00038

Sridharan, D., Levitin, D. J., and Menon, V. (2008). A critical role for the right fronto-insular cortex in switching between central-executive and default-mode networks. Proc. Natl. Acad. Sci. U.S.A. 105, 12569-12574. doi: 10.1073/pnas. 0800005105

Tully, L. M., Lincoln, S. H., Liyanage-Don, N., and Hooker, C. I. (2014). Impaired cognitive control mediates the relationship between cortical thickness of the superior frontal gyrus and role functioning in schizophrenia. Schizophr. Res. 152, 358-364. doi: 10.1016/j.schres.2013.12.005

Vivekananda, S. (2001). [1907], Complete Works of Swami Vivekananda, 9 Volumes, Kolkata, India: Advaita Ashrama ebooks. Available online at: https://ebooks. advaitaashrama.org/product/complete-works- of-swami-vivekananda-vol-1 (accessed June 12, 2021).

Wells, R. E., Kerr, C. E., Wolkin, J., Dossett, M., Davis, R. B., Walsh, J., et al. (2013a). Meditation for Adults with Mild cognitive impairment: a pilot randomized trial. J. Am. Geriatr. Soc. 61, 642-645. doi: 10.1111/jgs.12179

Wells, R. E., Yeh, G. Y., Kerr, C., Wolkin, J., Davis, R. B., Tan, Y., et al. (2013b). Meditation's impact on default mode network \& hippocampus in mild cognitive impairment: a pilot study. Neurosci. Lett. 556, 15-19. doi: 10.1016/j.neulet.2013. 10.001

Winblad, B., Palmer, K., Kivipelto, M., Jelic, V., Fratiglioni, L., Wahlund, L.-O., et al. (2004). Mild cognitive impairment-beyond controversies, towards a consensus: report of the International Working Group on Mild Cognitive Impairment. J. Intern. Med. 256, 240-246. doi: 10.1111/j.1365-2796.2004.01380.x

Wisse, L. E. M., Chételat, G., Daugherty, A. M., de Flores, R., la Joie, R., Mueller, S. G., et al. (2021). Hippocampal subfield volumetry from structural isotropic
$1 \mathrm{~mm}^{3}$ MRI scans: a note of caution. Hum. Brain Mapp. 42, 539-550. doi: 10.1002/hbm. 25234

Wong, W. P., Coles, J., Chambers, R., Wu, D. B.-C., and Hassed, C. (2017). The effects of mindfulness on older adults with mild cognitive impairment. J. Alzheimers Dis. Rep. 1, 181-193. doi: 10.3233/ADR170031

Yang, H., Leaver, A. M., Siddarth, P., Paholpak, P., Ercoli, L., St. Cyr, N. M., et al. (2016). Neurochemical and neuroanatomical plasticity following memory training and yoga interventions in older adults with mild cognitive impairment. Front. Aging Neurosci. 8:277. doi: 10.3389/fnagi.2016. 00277

Yang, H., Xu, H., Li, Q., Jin, Y., Jiang, W., Wang, J., et al. (2019). Study of brain morphology change in Alzheimer's disease and amnestic mild cognitive impairment compared with normal controls. Gen. Psychiatr 32:e100005. doi: 10.1136/gpsych-2018-100005

Yu, J., Rawtaer, I., Feng, L., Fam, J., Kumar, A., Cheah, I., et al. (2021). Mindfulness intervention for mild cognitive impairment led to attentionrelated improvements and neuroplastic changes: results from a 9-month randomized control trial. J. Psychiatr. Res. 135, 203-211. doi: 10.1016/j. jpsychires.2021.01.032

Zhao, H., Li, X., Wu, W., Li, Z., Qian, L., Li, S., et al. (2015). Atrophic patterns of the frontal-subcortical circuits in patients with mild cognitive impairment and Alzheimer's disease. PLoS One 10:e130017. doi: 10.1371/journal.pone.0130017

Conflict of Interest: The authors declare that the research was conducted in the absence of any commercial or financial relationships that could be construed as a potential conflict of interest.

Publisher's Note: All claims expressed in this article are solely those of the authors and do not necessarily represent those of their affiliated organizations, or those of the publisher, the editors and the reviewers. Any product that may be evaluated in this article, or claim that may be made by its manufacturer, is not guaranteed or endorsed by the publisher.

Copyright (c) 2021 Dwivedi, Dubey, Pansari, Bapi, Das, Guha, Banerjee, Pramanick, Basu and Ghosh. This is an open-access article distributed under the terms of the Creative Commons Attribution License (CC BY). The use, distribution or reproduction in other forums is permitted, provided the original author(s) and the copyright owner(s) are credited and that the original publication in this journal is cited, in accordance with accepted academic practice. No use, distribution or reproduction is permitted which does not comply with these terms. 